\title{
Magnetic Characterizations of Sol-Gel-Produced Mn-Doped ZnO
}

\author{
R. Asmatulu, ${ }^{1}$ H. Haynes, ${ }^{1}$ M. Shinde, ${ }^{1}$ Y. H. Lin, ${ }^{2}$ Y. Y. Chen, ${ }^{2}$ and J. C. Ho ${ }^{3}$ \\ ${ }^{1}$ Department of Mechanical Engineering, Wichita State University, Wichita, KS 67260, USA \\ ${ }^{2}$ Institute of Physics, Academia Sinica, Taipei 11529, Taiwan \\ ${ }^{3}$ Department of Physics, Wichita State University, Wichita, KS 67260, USA \\ Correspondence should be addressed to R. Asmatulu, ramazan.asmatulu@wichita.edu \\ Received 22 September 2010; Accepted 12 December 2010 \\ Academic Editor: Xuedong Bai
}

Copyright $\odot 2010$ R. Asmatulu et al. This is an open access article distributed under the Creative Commons Attribution License, which permits unrestricted use, distribution, and reproduction in any medium, provided the original work is properly cited.

Nanoparticles of $\mathrm{ZnO}$ doped with 6 at.\% Mn were produced by a sol-gel method. X-ray diffraction confirms the hexagonal structure as that of the parent compound $\mathrm{ZnO}$, and high-resolution electron transmission microscopy reveals a single-crystallite lattice. Magnetic measurements using a superconducting quantum interference device indicate that about one half of the Mn ${ }^{2+}$ ions follow Curie's law for paramagnetism. The remaining $\mathrm{Mn}^{2+}$ ions exhibit a weak ferromagnetic character, which might be induced through canted antiferromagnetic interactions.

\section{Introduction}

Wide-band gap semiconductor $\mathrm{ZnO}$ has a broad range of utilizations from ultraviolet protection to functional devices [1]. With a low-level substitution of $\mathrm{Zn}$ by certain magnetic cations, the generally categorized dilute magnetic semiconductors (DMSs) often exhibit ferromagnetism at ambient temperatures. After their discovery by Ohno et al. [2, 3], DMSs have attracted much attention in materials development towards spintronics application. Main emphases of the research so far appear to remain in the stage of processes evaluation in terms of magnetic behaviors of the products, while Toyoda et al. [4] have made self-interaction-correlated local-density approximation calculations on electronic structures for semiconductor spintronics. Beside the effort along this line, a sol-gel method was employed here to synthesize nanoparticles of $\left(\mathrm{Zn}_{0.94} \mathrm{Mn}_{0.06}\right) \mathrm{O}$ for comparison with other reported studies on $\mathrm{Mn}^{2+}$-doped zinc oxide including nanocrystals prepared by high-temperature hydrolysis [5] and films from plasma-assisted rf magnetron sputtering [6].

\section{Experimental}

All chemicals used were obtained from Sigma Aldrich unless otherwise specified. The relative amount of two precursors, $\mathrm{Mn}\left(\mathrm{NO}_{3}\right)_{2} \cdot 4 \mathrm{H}_{2} \mathrm{O}$ and $\mathrm{Zn}\left(\mathrm{NO}_{3}\right)_{2} \cdot 6 \mathrm{H}_{2} \mathrm{O}$, correspond to a $\mathrm{Mn} / \mathrm{Zn}$ atomic ratio of $0.06 / 0.94$. They were thoroughly mixed in deionized water on a hot plate with a magnetic stirrer. At 5-miunte intervals, benzoic acid, citric acid (Fisher Scientific), and ethylene glycol were subsequently added, each of the similar amount as that of zinc nitrate. The temperature was maintained at $30-40^{\circ} \mathrm{C}$ for complete dissolution. It was then raised and held at $70-80^{\circ} \mathrm{C}$ for 3 hours until all fluid was evaporated.

Evaporation left behind a viscous gel, which was transferred to an oven for dehydration at $150^{\circ} \mathrm{C}$ over a period of 15 hours. The hard, porous product was crushed and ground to fine powders and placed in a crucible for heattreatment at $550^{\circ} \mathrm{C}$ for two hours. Further regrinding yielded nanoparticles sample of $\left(\mathrm{Zn}_{0.94} \mathrm{Mn}_{0.06}\right) \mathrm{O}$.

\section{Results and Discussion}

A high-resolution transmission electron micrograph (TEM) in Figure 1 shows single-crystallites having a 30-nm average size. The crystalline lattice is clearly revealed in Figure 2. $\mathrm{X}$-ray diffraction (XRD) patterns in Figure 3 confirm the hexagonal wurtzite $(\mathrm{ZnS})$ structure of spatial $\mathrm{P}_{3} \mathrm{mc}$ group for $\mathrm{ZnO}$ [1], which has little change due to Mn doping up to 6 at.\%. This is not unexpected, considering the small difference between the ionic sizes of divalent $\mathrm{Zn}(0.74 \AA)$ and $\mathrm{Mn}(0.80 \AA)$ [5]. Presumably, $\mathrm{Mn}^{2+}$ enters the lattice substituting for $\mathrm{Zn}^{2+}$. However, one cannot be certain of 


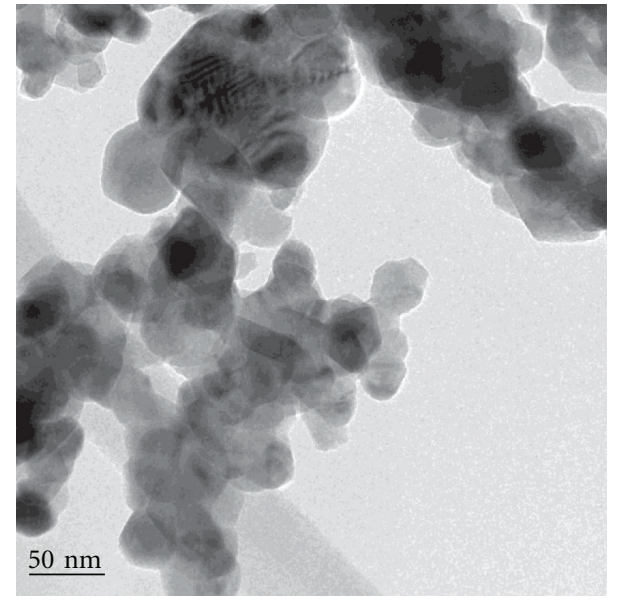

FIGURE 1: Transmission electron micrographs of nanocrystals in the $\mathrm{ZnO}-\mathrm{Mn}$ sample.

the actual distribution of $\mathrm{Mn}$ in lattice. The magnetic data analysis below could provide some information in this regard.

Magnetic behaviors are determined using a superconducting quantum interference device. Magnetization $M$, in units of emu/g with $\mathrm{g}$ referring to $1 \mathrm{~g}$ of sample, varies with temperature $T$ in Figure 4 at a constant field $H$ of 100 Oe. $M$ increases with decreasing temperature from $300 \mathrm{~K}$ to $5 \mathrm{~K}$, but it does not follow any simple fit to Curie's law for paramagnetic magnetization $M_{p}$

$$
\frac{M_{p}}{H}=\frac{N p^{2} \mu_{B}^{2}}{3 k_{B} T},
$$

where $N, p, \mu_{B}$, and $k_{B}$ are, respectively, the number of dipoles, effective moment for each dipole, Bohr magneton and Boltzmann constant. This is somewhat expected, since only uncoupled and non-interacting $\mathrm{Mn}^{2+}$ ions should yield a pure paramagnetic (PM) behavior. Other $\mathrm{Mn}^{2+}$ ions, if situated nearby to each other or in the form of clusters, could have ferromagnetic (FM) or antiferromagnetic (AFM) interactions among them. One way to observe these magnetic interactions is from the field dependence of magnetization at constant temperature. Consequently, magnetization data in Figure 5 were obtained at $300 \mathrm{~K}$, but with varying fields up to 6,000 Oe. A curvature is obvious below approximately 3,000 Oe.

Delineation of the total magnetization into components relies on the individual characteristic of each magnetic behavior. For PM, (1) prescribes a linear relation between $\mathrm{M}$ and $H$ at constant temperature. Ideal AFM would yield zero $M$ value. In contrast, for FM in single-domain nanoparticles, $M$ would increase initially with $H$ and reaches a saturation value at certain higher fields. Numerically, the delineation is successfully achieved as in Figure 4, with a paramagnetic magnetization $M_{p}=5.3 \times 10^{-6} \mathrm{Hemu} / \mathrm{g}$ and a ferromagnetic magnetization which saturates at $M_{s}=$ $0.0068 \mathrm{emu} / \mathrm{g}$ near 3,000 Oe.

There are a total of $4.5 \times 10^{20} \mathrm{Mn}^{2+} / \mathrm{g}$ in $\left(\mathrm{Zn}_{0,94} \mathrm{Mn}_{0.06}\right) \mathrm{O}$. By taking the often quoted effective moment $p=5.9 \mu_{B}$ for

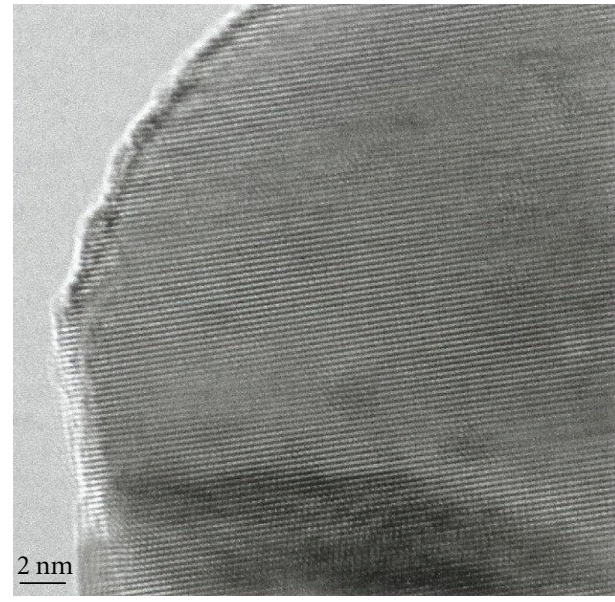

FigURE 2: High-resolution transmission electron micrograph revealing the crystal lattice.

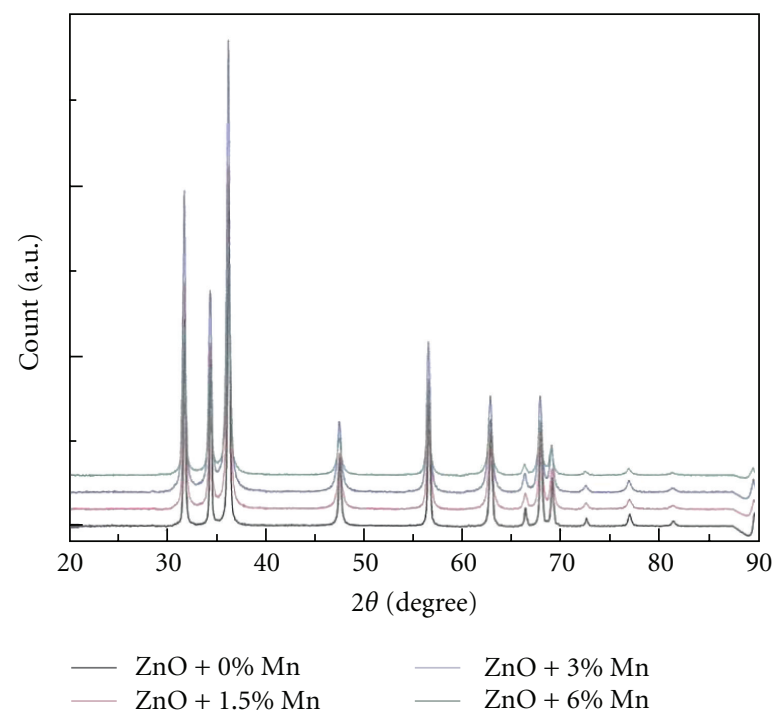

Figure 3: X-ray diffraction patters remain practically the same for $\mathrm{Mn}$ additions up to 6 at. $\%$.

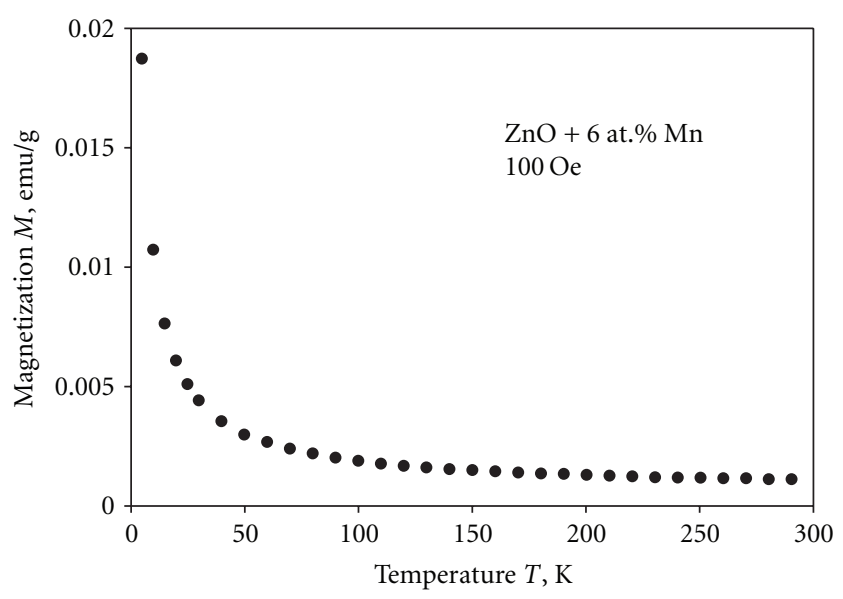

FIGURE 4: Magnetization increases with decreasing temperature, but does not follow the Curie's law for a paramagnet. 


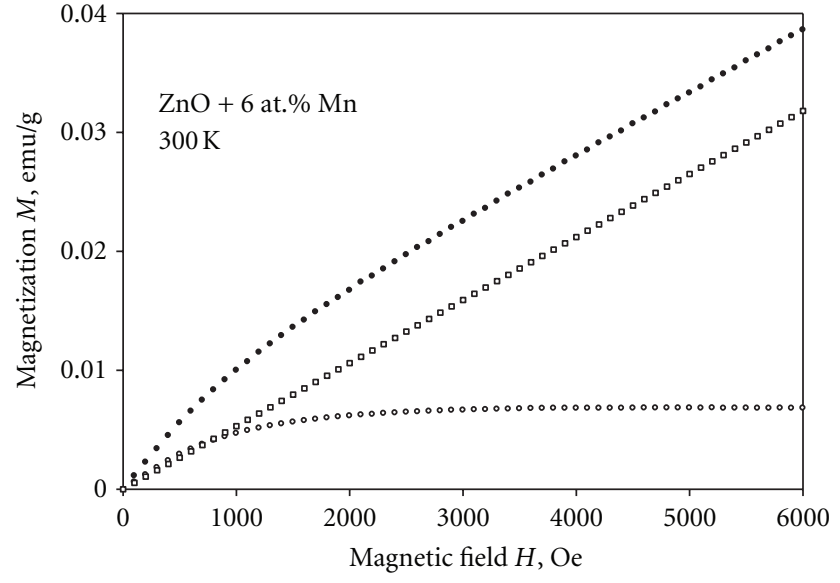

FIGURE 5: Experimentally obtained magnetization (close circle) can be delineated into a paramagnetic contribution (open square) following (1), and a ferromagnetic-type contribution (open circle), which increases with increasing field to saturation near 3,000 Oe.

each non-interacting $\mathrm{Mn}^{2+}$ ion, the experimentally obtained $M_{p}$ corresponds to $N=2.2 \times 10^{20} \mathrm{Mn}^{2+} / \mathrm{g}$ in (1). In other words, about one half of $\mathrm{Mn}^{2+}$ ions appear to exist as PM and the remaining $2.3 \times 10^{20} \mathrm{Mn}^{2+} / \mathrm{g}$ would fall in the magnetically interacting categories. In principle, one could derive the number of FM ions from $M_{s}$ and $5 \mu_{B} / \mathrm{Mn}^{2+}$. If so, there would be only $1.5 \times 10^{17}$ or $0.03 \%$ of the total $\mathrm{Mn}^{2+} / \mathrm{g}$ in this state. Ferromagnetism could prevail through Zener's double exchange mechanism [7]. Another source of $M_{s}$ could be based on that magnetic interactions among close-by neighboring $\mathrm{Mn}^{2+}$ ions in oxides are more likely antiferromagetic in nature through superexchange. Under certain magnetically frustrated conditions, it is possible to have a canted AFM, which would result in a small net moment or a weak FM as observed here. Such a scenario is corroborated by electron spin resonance (ESR) experiments. The ESR intensity is enhanced as the temperature drops from $300 \mathrm{~K}$ to $77 \mathrm{~K}$, signifying AFM. Meanwhile, a spin $g$ value of 2.012 was also obtained. It corresponds to a weak ferromagnetism.

$\mathrm{Yu}$ et al. [6] reported a similar $\mathrm{M}-\mathrm{H}$ curve in a $\left(\mathrm{Zn}_{0.99} \mathrm{Mn}_{0.01}\right) \mathrm{O}$ film, showing a paramagnetic character with weak ferromagnetism behavior. Even after nitrogen incorporation to enhance the ferromagnetic signal, the saturation magnetization is only $0.5 \mu_{\mathrm{B}} / \mathrm{Mn}^{2+}$, an order of magnitude smaller than the free $\mathrm{Mn}^{2+}$ value. Meron and Markovich [5] also pointed out the difficulty in estimating the percentage of ferromagnetic $\mathrm{Mn}^{2+}$ ions in their $\left(\mathrm{Zn}_{1-x}\right.$ $\left.\mathrm{Mn}_{x}\right) \mathrm{O}$ nanocrystals. Using a similar type of analysis as given above, they first calculated the paramagnetic part in the sample. Relying on the slope of a linear region at high fields in an $\mathrm{M}(\mathrm{H})$ plot, instead of delineation of the data as in Figure 5, they arrived at an upper limit of $40 \%$ for the magnetically interacting $\mathrm{Mn}^{2+}$. It is also comparable to the value obtained in this work. Beyond that, however, a notable difference exists between the current result and that of Meron and Markovich [5] in the magnitude of saturation magnetization. One plausible explanation for our much smaller $M_{s}$ may be due to a particle size effect. As particle size decreases, the percentage of spins on surface would certainly increase. These spins tend to generate strong magnetic frustration, which would enhance canted antiferromagnetism, thus the ferromagnetic-like behavior. Indeed, the average particle size in this study is nearly an order of magnitude larger than those of the earlier study [5].

\section{Conclusions}

In the present study, sol-gel-based $\left(\mathrm{Zn}_{0.94} \mathrm{Mn}_{0.06}\right) \mathrm{O}$ nanoparticles were synthesized using a sol-gel process and their magnetic properties were investigated. It was determined that the nanoparticles have roughly equal amount of $\mathrm{Mn}^{2+}$ ions in either paramagnetic or magnetically interacting states. The latter is mainly antiferromagnetic in nature, but magnetic frustration causes part of them to exhibit a canted AFM, which results in a ferromagnetic-like behavior. These doped nanomaterials may be used in various applications, such as electronic, sensor, spintronic, coating, and solar and fuel cells.

\section{Acknowledgments}

The work at Academia Sinica was supported by the National Research Council, the Republic of China, under Grant no. NSC 97-2120-M-001-007. The authors would also like to thank Professor J.G. Lin of National Taiwan University for ESR measurements.

\section{References}

[1] B. S. Barros, R. Barbosa, N. R. dos Santos, T. S. Barros, and M. A. Souza, "Synthesis and $\mathrm{x}$-ray diffraction characterization of nanocrystalline $\mathrm{ZnO}$ obtained by Pechini method," Inorganic Materials, vol. 42, no. 12, pp. 1348-1351, 2006.

[2] H. Ohno, H. Munekata, T. Penney, S. Von Molnar, and L. L. Chang, "Magnetotransport properties of p-type (In,Mn)As diluted magnetic III-V semiconductors," Physical Review Letters, vol. 68, no. 17, pp. 2664-2667, 1992.

[3] H. Ohno, A. Shen, F. Matsukura et al., "(Ga,Mn)As: a new diluted magnetic semiconductor based on GaAs," Applied Physics Letters, vol. 69, no. 3, pp. 363-365, 1996.

[4] M. Toyoda, H. Akai, K. Sato, and H. Katayama-Yoshida, "Electronic structures of (Zn, TM)O (TM: V, Cr, Mn, Fe, Co, and Ni) in the self-interaction-corrected calculations," Physica B: Condensed Matter, vol. 376, no. 1, pp. 647-650, 2006.

[5] T. Meron and G. Markovich, "Ferromagnetism in colloidal Mndoped ZnO nanocrystals," Journal of Physical Chemistry B, vol. 109, no. 43, pp. 20232-20236, 2005.

[6] Z. Tiejun, C. Zexiang, and T. Bo, "Nonlinear optical property of aligned multi-walled carbon nanotube film," in Proceedings of the Symposium on Photonics and Optoelectronics (SOPO '09), August 2009.

[7] K. Sato, L. Bergqvist, J. Kudrnovský et al., "First-principles theory of dilute magnetic semiconductors," Reviews of Modern Physics, vol. 82, no. 2, pp. 1633-1690, 2010. 

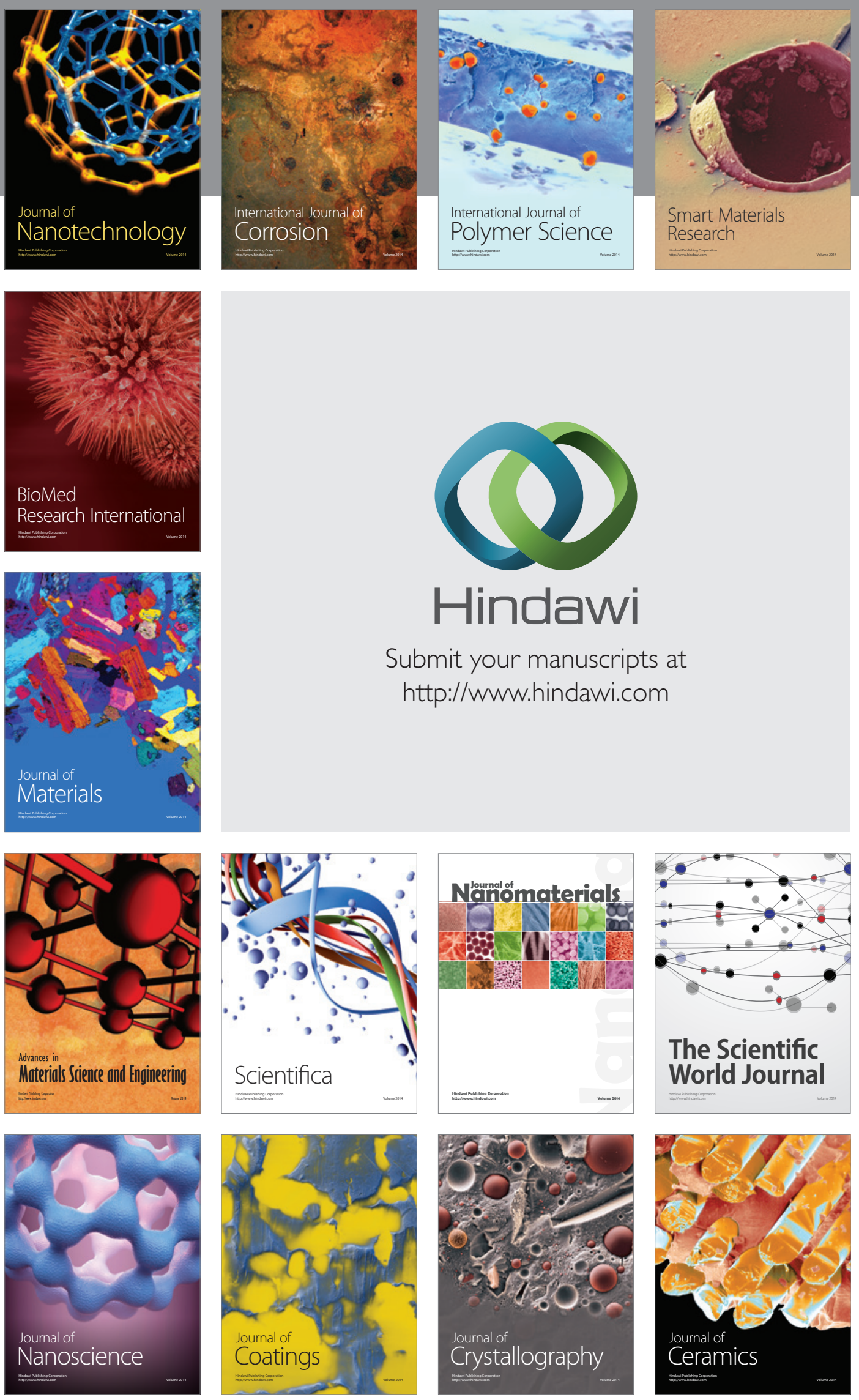

The Scientific World Journal

Submit your manuscripts at

http://www.hindawi.com

\section{World Journal}

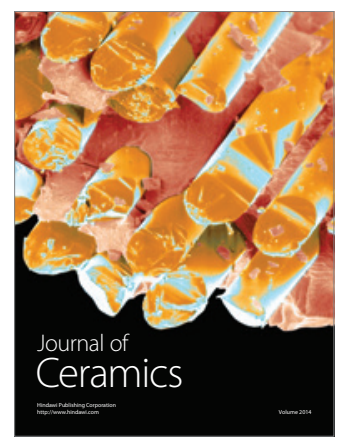

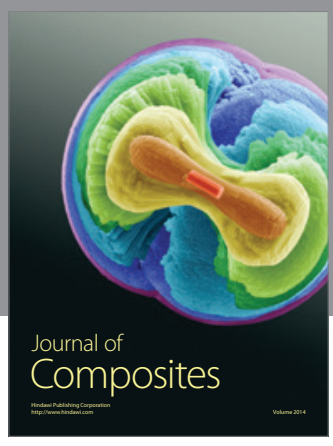
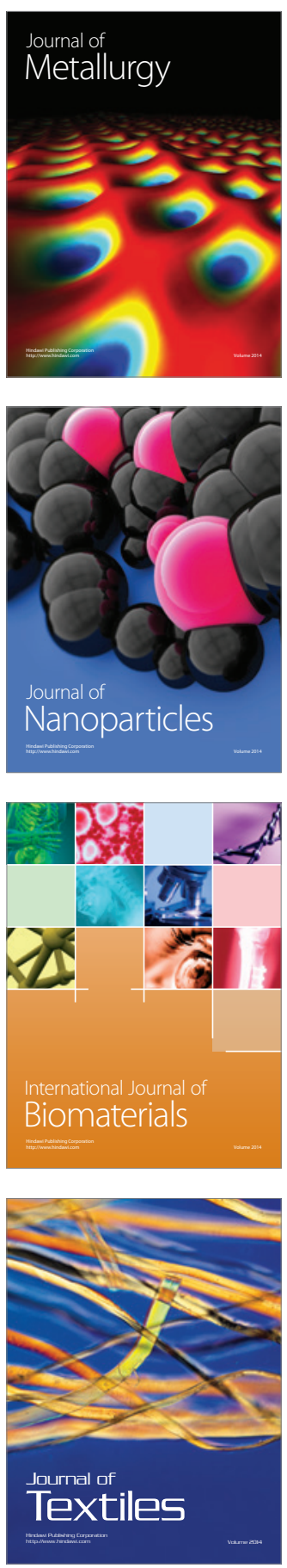